\title{
Pipe Friction Parameters Identification of Heat-supply Networks
}

\author{
Yue Xingzuo \\ Wuhan institute of shipbuilding technology \\ wuhan china \\ 84771162@qq.com
}

\begin{abstract}
The central heating system is an important infrastructure of city, because it has the advantages of energy saving, environmental protection and the quality of heat supply and so on, will be more widely used in the "energy-saving emission reduction" in the environment. Heat supply network is an important part of centralized heating system, with a large scale, complex structure, huge investment and social impact of major characteristics, so much concern. At present, more research, hydraulic calculation of heating network operation scheduling and reliability analysis in several aspects, and heating pipe network resistance characteristics identification research is less. If unable to accurately obtain the actual operation of heating pipe network resistance coefficient under the condition of hydraulic condition, heating pipe network calculation and operation scheduling are only stay in theoretical analysis and qualitative simulation. Calculation and analysis of the results are often inconsistent with the actual operation, thereby greatly reducing the research results and practical value. At present, for the study of the pipe friction parameters of the few, the research of heating network identification model is still relatively small, single and identification method, identification accuracy degree is not high, the identification of time-consuming calculation. Therefore, in-depth study of the heating pipe resistance coefficient identification problem has important theoretical significance and practical value.
\end{abstract}

Keywords-heating pipe network; identification of drag coefficient matrix theory; observation points; optimal layout; relative sensitivity

\section{INTRODUCTION}

In the study of heating pipe resistance coefficient identification problem of time due to the actual observation conditions of the existing heating network generally can not meet the requirements of the identification of observability, combining the theoretical analysis and the generalized inverse matrix theory of solutions of linear equations, and puts forward a method of generalized inverse matrix theory identification for heat pipe resistance coefficient based on. Study on resistance coefficient identification problem of heating pipe network of the method can be applied to general observation conditions, which solve the identification equations iterative method gradually linearized by using generalized inverse solution to equations, and the resistance in the coefficient as the identification results, the actual value of the drag coefficient approximation of heating pipe network. Then, the application method of generalized inverse matrix theory of identification of heating pipe network resistance coefficient based on identification examples.
In may obtain more observational data conditions, can use part tube section (usually a group of link pipe resistance coefficient of the initial value) as the known condition identification, identification of heating pipe resistance coefficient is obtained. Considering the use of space heating pipe network model can more directly reflect the actual situation of the heating pipe network, using the block matrix representation of space heating pipe resistance coefficient identification problem, chain pipe selection scheme in solving the identification problem at the same time, according to the characteristics of heating pipe network is proposed for the space heating pipe resistance coefficient identification.

Combined with the clustering analysis of the theory put forward section flow and node pressure method of optimal layout of observation points reflect the change of heating network pipe resistance coefficient based on. Matrix in matrix theory of matrix differential operation principle and heating pipe resistance coefficient change on the linear effect of pipe section flow and node pressure based on the analytical results, on the basis of the above study, proposed the use of pipe flow and node pressure observation point relative sensitivity studies the problem of optimal layout of observation points, and according to the heating pipe network design value calculation of relative sensitivity. With the method of the inverse matrix theory to identify heating pipe resistance coefficient, to identify the equations of generalized inverse solution as the heating pipe resistance coefficient identification results. In have more perfect observing conditions, can use the identification method of numerical a group of link pipe resistance coefficient based on more close to the actual value of the heating pipe resistance coefficient identification results.

At present, the heating pipe network system simulation, the heating network hydraulic condition analysis, and heating pipe network reliability and fault condition calculation analysis and other aspects of the simulation, analysis and calculation in theory, the results of the research most stay and qualitative, and applied in the practical engineering, the calculation and analysis of the results are often inconsistent with the actual results, so may affect the research results of the practical value. Lead to the calculation results with the actual measured value between operation causes the deviation is more, summarized mainly has the following several.

(1) the basic data is accurate: heating pipe network system models are required to calculate the large amount of data, but also need to go through the gradual induction, statistics, analysis and processing, therefore, get closer to the actual value of the basic data needs a lot of research 
work. This will give access to the resistance value bring some inconvenience.

(2) pipe resistance coefficient uncertainty: changes due to pipe segment flow capacity due to various internal and external factors, so the introduction of coefficient of section resistance characteristics of a characterization of pipe in pipe resistance loss calculation formula, called the pipe resistance coefficient, to table collection section resistance. Due to the changes of the laying of pipe resistance coefficient by various influence factors of age, diameter, pipe, conveying water and pipe inner wall corrosion, leading to resistance coefficient is difficult to determine accurately calculated by the formula.

(3) the observation and control equipment can not reach the heating pipe network operating parameters to determine the required conditions: automatic observation and control level of the existing heating network than in the past have been greatly improved, the number of heating pipe in the heat source and the thermal station provided with observation and control system in the continuous increase, but in the heating pipe network installed observation and other nodes the control system has basically no, this leads to only a portion of the heating pipe network operating parameters is known, to determine the effect of pipe resistance coefficient.

(4) error equipment observations: even for those who have automatic observation and control system of more perfect heating pipe network, since any observation equipment with the instrumental errors still exist for gross error and random error of human, which also leads to the observed data to a certain extent from the pipe itself value.

There are many factors that affect the resulting in the deviation of the theoretical calculations and the measured data, as a parameter to characterize the heating pipe network resistance characteristics of pipe resistance coefficient is accurate, will directly affect the accuracy of the calculation results of heating pipe network hydraulic. Calculation of heating network system simulation and hydraulic conditions in the previous analysis is built based on the numerical results of the heating pipe resistance coefficient design value, the calculation of this condition often results and practical operation value are very different, so if the numerical can through the parameter identification method of identification theory to obtain the actual running of the resistance of the heating pipe network system, the existing heating network system simulation, hydraulic regime calculation analysis, operation scheduling, fault condition calculation analysis and traffic accident treatment of utility will get to improve.

\section{THE USE OF MULTIPLE HYDRAULIC LOADING} IDENTIFICATION HEATING PIPE RESISTANCE COEFFICIENT

The calculation results based on the hydraulic conditions on the existing network of heating, identification of drag coefficient can be observational studies. In the analysis and come to meet the basic necessary conditions for observation of heating pipe network in identification of drag coefficient of energy, and then put forward the judgment of heating pipe network in identification of drag coefficient whether meet the conditions determining the observation of energy, and can satisfy the pipe friction parameters observed under conditions of method of solving questions asked.
Heating pipe network hydraulic condition characteristic rules can calculate the said equation with the hydraulic condition, the foundation of these equations is heating pipe resistance coefficient identification. This section firstly based on graph theory and matrix theory will reflect a series of equations combined hydraulic conditions of heating pipe network calculation and identification of drag coefficient expression into the matrix equations of the form.

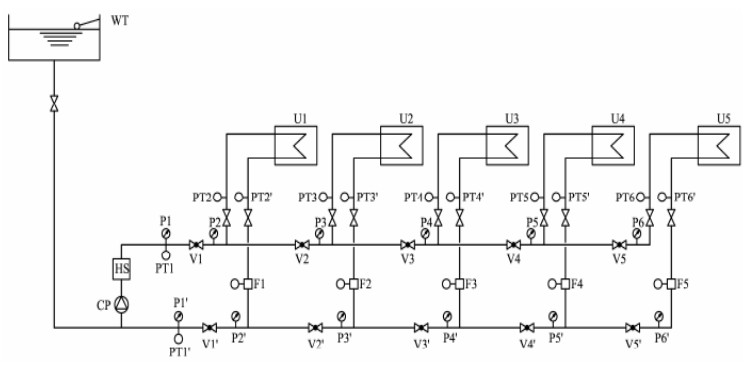

Figure 1. System diagram of the multi-function experiment platform

Equations form by using heating pipe resistance coefficient identification equations and hydraulic regime calculation is the same, but the known quantity and unknown amount of them is different. As mentioned earlier, the hydraulic conditions of heating pipe network is in the calculation of drag coefficient as known quantity under the condition of the solution of equations of pipe flow and node pressure value. As the number of unknown variables included in this case the set of equations is exactly equal to the number of equations, so the hydraulic condition calculation equations have a unique solution. And the heating pipe network in identification of drag coefficient is in the part of pipe flow and node pressure value is known, solving the equations in resistance coefficient and other pipe flow and node pressure process segment value, because the number of unknown quantity contained in this case equations in general higher than the number of equations, therefore the drag coefficient identification equations in general no unique solution. Identification method is studied in this paper is actually based on the part of pipe network operation parameters (pipe flow and node pressure) observations estimation method of resistance coefficient.

From the mathematical analysis of heating pipe network in identification of drag coefficient of observability problem is actually the relationship between quantity comparison equations in the number of equations and unknowns, that is to say, observability condition is to ensure that the conditions in each of the equations are independent of each other. Because very few study on correlation of nonlinear equations by means of the control theory in the aspects of the relevant theoretical research of heating network identification of drag coefficient of observability problem

\section{A. Heating pipe network in identification of drag coefficient of determination and solution}

Heating pipe resistance coefficient identification problem actually belongs to the category of system parameters identification in control theory. Results observation of using the system in control theory, can discuss observations and analysis of the heating pipe resistance coefficient identification problem. 
Before solving the heating pipe resistance coefficient identification problem, need to determine whether the heating pipe network research and observation data meet the observation condition identification. First determine whether a heating pipe network and the observation data to satisfy the identification of observability, and then the heating pipe resistance coefficient identification solution.

Identification of drag coefficient of heating pipe network on the basis of the model, is actually a node to estimate the resistance coefficient of flow and pipe flow observation data using a network node pressure, and equations is also in the part of pipe network operation parameters under the condition of known solution. Heating pipe resistance coefficient identification equations for general viewing conditions is not the existence and uniqueness of solution, and in the identification of observability can meet the conditions can be obtained by solving equation group is the only the pipe resistance coefficient. To meet the heating pipe resistance coefficient identification observability conditions, can solve the multi process identification of hydraulic conditions summarized as the following 3 steps:

(1) according to the ratio of multiple hydraulic conditions calculated for each pipe segment in different hydraulic conditions flow ratio;

(2) to calculate the pipe flow hydraulic conditions of simultaneous multiple hydraulic conditions;

(3) each pipe flow equation of the hydraulic conditions, can be calculated by the numerical value of each pipe resistance system.

In the heating pipe network in identification of drag coefficient in the process of calculation, considering the large amount of calculation of heating pipe network identification may be relatively large, this section in the direct solution based on a calculation method can reduce the calculation dimension presents a indirect and calculation method, so as to improve the identification efficiency calculation.

\section{IDENTIFICATION METHOD OF NUMERICAL SECTION} RESISTANCE LINE

Combined with the analysis and the generalized inverse theory of solution of linear equations of the proposed identification method, coefficient of heating pipe network resistance based on the theory of generalized inverse matrix. The method can be used for all nodes can be observed and a small amount of pressure pipe flow can be heating pipe resistance coefficient identification problem of observation conditions. While the actual heating pipe network in general only part of the nodes and pipe arranged pressure and flow measuring device, in order to solve the heating pipe resistance coefficient identification problem under this condition, this chapter puts forward the method of generalized inverse matrix theory of heating pipe network based on the identification of resistance coefficient, the generalized inverse solution to solve the identification equations an iterative method of stepwise linearization using the numerical solution, and to the resistance of the system as a result of identification.

Finally, the above identification method is applied to the heating pipe resistance coefficient identification example, process and result of calculation indicate: recognition under general viewing conditions, compared with the existing heating pipe resistance coefficient identification method, identification coefficient of heating pipe network resistance identification method based on the theory of generalized inverse matrix has high computation efficiency and is closer to the real value (count example numerical setting resistance system) identification results. The method is to solve the identification method of the actual heating pipe network of observation points less, observation data lack conditions and means. In with other observation conditions, can further study the heating pipe resistance coefficient identification problem.

The actual observation conditions in the existing heating network, identification of observability conditions is generally not able to meet, at this time, heating pipe resistance coefficient identification method of heating pipe network is actually a kind of drag coefficient estimation. In the observation conditions, in order to get closer to the actual value of the heating pipe resistance coefficient identification results, known as identification condition identification method using a group of link pipe resistance coefficient of initial value. The method can identify conditions in the rich conditions, that is, a group of link pipe resistance coefficient estimates can be obtained, and all nodes pressure can be observed under the conditions of direct calculation, and determine all numerical section resistance system. Therefore, the method is efficient.

In the analysis of heating pipe network in identification of drag coefficient of sensitivity, hold the tube changes the drag coefficient may cause changes in pipe flows and node pressure pipe heating pipe, heating pipe network when the drag coefficient of a pipe segment changes, will cause the pressure of each node in the heating networks and the pipe flow changes, but it is not as for the influence degree of each node pressure or each pipe segment flow. In the sensitivity analysis, the assumption that the presence of small pipe resistance coefficient under the condition of the change, the node of large pressure variation may be better observed location layout of pressure; similarly, a great changing flow pipe segment may be a better position arrangement of traffic observation point. Using the identification equation, and according to the matrix the operational rule of differential expressed sensitivity matrix flow and pressure on the drag coefficient of the heating pipe network. Analysis on the effect of pipe flow and node pressure observation period value of the drag coefficient of variation of heating pipe network. Find more can reflect changes in the size of the drag coefficient of the observation point, that the more sensitive observation point. The study found that heating pipe resistance coefficient identification problem, the majority of the nodes relative pressure value is relative to the flow value is more sensitive. But the flow of observation point selection, changes in pipe flow is smaller for the pipe resistance in the heating pipe network in the whole number is sensitive.

\section{CONCLUSION}

Matrix analysis theory of heating network identification of drag coefficient was studied based on relevant problems. The main conclusions are as follows:

(1) the establishment of the form of expression in heating network identification of drag coefficient matrix equation. Combined with the system observability theory, and points out that the heating pipe network node pressure 
can be observed is the necessary condition to meet the observability of heating pipe network resistance coefficient identification, and proposes a satisfied identification can determine conditions and identification method of solving observability, provides a new research method for the theoretical research and experimental test of heating pipe network identification of the resistance coefficient. To meet the heating pipe resistance coefficient identification observability condition, used to meet the heating pipe network resistance coefficient identification can identify the observation condition calculate can greatly improve the computational efficiency of numerical identification, get closer to the real results of the drag coefficient.

(2) consider the actual observation conditions of the existing heating network identification of drag coefficient can not meet the general identification of observability requirements, combined with the analysis of theory and the generalized inverse matrix theory of solutions of linear equations, put forward the theory of generalized inverse of matrices of the heating pipe friction parameters identification method based on. Heating pipe resistance coefficient identification problem of the method can be applied to general observation conditions, which solve the identification equations in an iterative method of successive linearization using generalized inverse solution with numerical solution, and the resistance of the system as a result of identification. The identification is based on the application of heating pipe network resistance coefficient identification method of generalized inverse matrix theory in general observation condition of the examples, the results show that: in general observation conditions, coefficient of heating pipe network resistance identification method of generalized inverse matrix theory which is based on identification of high computation efficiency and is more close to the actual resistance heating pipe network numerical system identification results.

(3) provide more identification known conditions in the case of be, use a set of numerical studies link pipe resistance identification method known as identification system based on conditions of heating pipe network resistance coefficient identification problem solving. Each node of the pressure can be observed and a group of link pipe resistance coefficient can be obtained under conditions can be directly calculated and determined all numerical section resistance system, in which the choice of pipe resistance of vector norm minimum values as the identification results, this method has high computing efficiency. Considering the use of space heating pipe network model can more accurately reflect the actual situation of heating pipe network, this paper introduces the hydraulic condition of space heating pipe network computation, using block matrix representation problem of identification, identification method and combining the characteristics of space heating network is proposed for the space heating network. On the basis of this, the above identification methods are applied to the plane and space heating pipe resistance coefficient identification example, identification of the calculation results show that the superiority of the identification method.

(4) combined with clustering analysis theory, put forward the heating network identification of drag coefficient of observation point relative sensitivity and the concept of optimization method based on the observation layout section flow and node pressure tube reflect the change of heating pipe network resistance coefficient, and according to the heating pipe network design value gives the matrix expression form, make the observation point of the optimal layout scheme more reasonable. This model is applied to a numerical example, the results showed that the relative sensitivity of node pressure is generally higher than that of the relative sensitivity of pipe flow; and the flux observation point selection, pipe flow is small random variation for each pipe section number more sensitive resistance.

\section{REFERENCES}

[1] Yongxin Liu, Pinghua Zou. Pipe Resistance Identification of an Experimental Heat-supply Network based on Minimal Norm and GA Methods [C]. Proceedings of Third International Conference on Information and Computing Science, Wuxi, Jiang Su, China, 4-6 June 2010: 50-53J.

[2] Qian W N, Zhou A Y. Analyzing Popular Clustering Algorithms from Different Viewpoints [J]. Journal of Software, 2002, $8: 1382-1394$

[3] Zhang R. Research on the Data Clustering Techniques [J]. Computer Engineering and Applications, 2002, $16: 145-170$.

[4] Zhang X D. Matrix Analysis and Applications [M]. Beijing : Tsinghua University Press, $2004: 85-95$

[5] Falco D D, Pennestri E, Vita L. Investigation of the Influence of Pseudoinverse Matrix Calculations on Multibody Dynamics Simulations by Means of the Udwadia-Kalaba Formulation [J]. Journal of Aerospace Engineering, 2009, 22(4) : 365-372.

[6] Mbekhta M, and Suciu L. Generalized Inverses and Similarity to Patial Isometries [J]. Journal of Mathematical Analysis and Applications, 2011, Impress, Accepted Manuscript.

[7] Sankaran S G, and Beex A A. Convergence Behavior of Affine Projection Algorithms [J]. IEEE Trans Signal Processing. 2000, 48(4) : 1086-1096.

[8] Pasha M F. Uncertainty Analysis and Calibration of Water Distribution Quality Models [D]. Arizona : Dissertation for the Doctoral Degree in the University of Arizona, 2006 : 86-91.

[9] Kapelan Z S, Savic D A, Walters G A. Calibration of Water Distribution Hydraulic Models Using a Bayesian-Type Procedure [J]. Journal of Hydraulic Engineering, 2007, 133(8) : 927-936.

[10] Ainola L, Koppe T, Tiiter K. Water Network Model Calibration Based on Grouping Pipes with Similar Leakage and Roughness Estimates [C]// Proceedings of Joint Conference on Water Resources Engineering and Water Resources Planning \& Management. Minneapolis : ASCE, $2000: 50-56$ 